\title{
Theory and application of general linear image processing
}

\author{
Jean-Charles Pinoli ${ }^{1 *}$, Guang Deng ${ }^{2}$ and Karen Panetta ${ }^{3}$
}

Image modeling in terms of physical/mathematical formation and the human visual system plays an important role in developing new image processing techniques. Parameters such as scale, orientation, texture, morphology, and color must be taken into consideration in order to solve complicated image processing problems efficiently. Although the classical linear image processing (CLIP) approach has played a central role in image processing, it is not necessarily the best and even the right choice. General linear image processing (GLIP) approaches based on different image modeling techniques have been studied to overcome some of the problems associated with CLIP. Indeed, using general linear algebra, it is possible to develop entirely new general linear operators and transforms. It is even possible to define entirely new general linear operations (addition, scalar multiplication, convolution, etc.) in order to describe how images are combined, amplified, transformed, analyzed, compared, and measured.

There have been 24 articles submitted to this special issue. After two rounds of peer review process, five articles have been selected.

In the first article, the authors propose a generalization of the logarithmic image processing (LIP) model by introducing several parameters into the model. The LIP model, which is based on a physical image formation model, appears as a special case of the proposed model when these parameters are set to specific values. New generalized operations are defined. The application of the proposed model is demonstrated by solving image fusion problems in a multi-resolution framework.

In the second article, the authors present a blind watermarking scheme in which the watermark is embedded in the wave atom transform domain. The wave atom transform, which is a relatively new mathematical representation of images, has been demonstrated to produce sharper frequency localization than filter bank-based

\footnotetext{
* Correspondence: pinoli@emse.fr

'Ecole Nationale Supérieure des Mines de Saint-Etienne, CIS-LPMG/CNRS,

Saint-Etienne Cedex 2, France

Full list of author information is available at the end of the article
}

wavelet packets. The authors show that using the wave atom representation the proposed watermarking algorithm is robust against various attacks.

In the third article, the authors present methods for meeting the challenges of using mobile projectors ubiquitously in the same manner as mobile phones. A visionbased projector-camera (i.e., pro-cam) system is often the choice for researchers in this field because it allows object augmentation everywhere without relying on separate tracking hardware. Furthermore, a user can interact directly with the projection. However, there are challenges encountered when making a pro-cam system aware of its surrounding objects, while still retaining ubiquity. One issue is that ubiquitous projection implies use in an unknown environment that has not been prepared. The second problem, called "projective interference", can lead to false detections of the environment and object. The authors present solutions using nonintrusive projection, which can ensure correct vision-based analysis. The external appearances of the environment and object are hardly changed by the overlaid projected content. Finally, an unsupervised visual detector is used to guarantee robustness of the detection regardless of lighting variations. Several image processing algorithms are applied in the framework including image warping using perspective transformation, color conversion using the nearestneighbor search algorithm, and multiscale visual detection.

A persistent issue in image processing is quantifying the improvement or measuring the visual quality resulting from the output image generated by an image processing algorithm. The fourth article deals with utilizing and deriving new quantitative metrics that leverage on the well-known structural similarity (SSIM) indexes. The authors show that SSIM index, which is used in image processing to assess the similarity between an image representation and an original reference image, can be formulated as a locally quadratic distortion measure. In addition, the authors derive the high-resolution SSIMRate-Distortion Function (RDF) and provide a simple 
method to numerically compute an approximation of the SSIM-RDF of real images.

Finally, the transmission of images requires quality compression algorithms that maintain the most pertinent image details. The fifth article presents a new block-based image compression scheme based on generation of fixed block sets, called classified energy blocks and classified pattern blocks. The method shows promise for a high compression ratio and acceptable image quality in terms of PSNR levels even at low bit rates. The encoding parameters are the block scaling coefficient and the index numbers of energy and pattern blocks (IE, IP) determined for each block of the input images. In the decoding process, all these parameters are sent through the communication channel from the transmitter part to the receiver part and the classified energy and pattern blocks (CEPB) associated with the index numbers are pulled from the CEPBs. Then, the blocks of the input image are reconstructed in the receiver part using the presented mathematical model. In this study, in order to remove the blocking effect and improve the PSNR levels, a post-processing filter is used on the reconstructed images and the PSNR levels are improved in the range of $0.5-1 \mathrm{~dB}$. The speed of the algorithm and the compression ratio are also increased by adjusting the size of the CEPB using an efficient clustering algorithm.

\section{Acknowledgements}

As guest editors, we would like to express our sincere gratitude to all those who have contributed to this special issue, and the reviewers who have provided many critical comments and constructive suggestions to the manuscripts submitted. We are sorry that many of the articles submitted are not able to be included in this special issue due to the time constraints and the amount of modification required. We do hope that this special issue will boost the interest of GLIP within the image processing and computer vision community.

Jean-Charles Pinoli

Guang Deng

Karen Panetta

\section{Author details}

${ }^{1}$ Ecole Nationale Supérieure des Mines de Saint-Etienne, CIS-LPMG/CNRS, Saint-Etienne Cedex 2, France ${ }^{2}$ Department of Electronic Engineering, La Trobe University, Bundoora, VIC 3086, Australia ${ }^{3}$ Department of Electrical and Computer Engineering, Tufts University, Medford, MA, USA

Received: 4 April 2011 Accepted: 7 February 2012 Published: 7 February 2012

doi:10.1186/1687-6180-2012-21

Cite this article as: Pinoli et al:: Theory and application of general linear image processing. EURASIP Journal on Advances in Signal Processing 2012 2012:21.

\section{Submit your manuscript to a SpringerOpen ${ }^{\mathcal{O}}$ journal and benefit from:}

- Convenient online submission

- Rigorous peer review

- Immediate publication on acceptance

- Open access: articles freely available online

- High visibility within the field

- Retaining the copyright to your article

Submit your next manuscript at $\gg$ springeropen.com 